\title{
Proteomics of Porphyromonas gingivalis within a model oral microbial community
}

\author{
Masae Kuboniwa ${ }^{1}$, Erik L Hendrickson ${ }^{2}$, Qiangwei Xia ${ }^{2,3,6}$, Tiansong Wang ${ }^{2,3}$, \\ Hua Xie ${ }^{4}$, Murray Hackett ${ }^{2}$ and Richard J Lamont*5
}

Address: ${ }^{1}$ Department of Preventive Dentistry, Osaka University Graduate School of Dentistry, Osaka, Japan, ${ }^{2}$ Department of Chemical Engineering, University of Washington, Seattle, WA, USA, ${ }^{3}$ Department of Microbiology, University of Washington, Seattle, WA, USA, ${ }^{4}$ School of Dentistry, Meharry Medical College, Nashville, TN, USA, ${ }^{5}$ Department of Oral Biology, University of Florida, Gainesville, FL, USA and ${ }^{6}$ University of Wisconsin-Madison, Department of Chemistry, Madison, WI, USA

Email: Masae Kuboniwa - kuboniwa@dent.osaka-u.ac.jp; Erik L Hendrickson - elh@u.washington.edu; Qiangwei Xia - qxia@chem.wisc.edu; Tiansong Wang - tswang@u.washington.edu; Hua Xie - hxie@mmc.edu; Murray Hackett - mhackett@u.washington.edu;

Richard J Lamont* - rlamont@dental.ufl.edu

* Corresponding author

Published: 19 May 2009

BMC Microbiology 2009, 9:98 doi:10.1 186/147/-2180-9-98
Received: 30 January 2009

Accepted: 19 May 2009

This article is available from: http://www.biomedcentral.com/I47I-2/80/9/98

(c) 2009 Kuboniwa et al; licensee BioMed Central Ltd.

This is an Open Access article distributed under the terms of the Creative Commons Attribution License (http://creativecommons.org/licenses/by/2.0), which permits unrestricted use, distribution, and reproduction in any medium, provided the original work is properly cited.

\begin{abstract}
Background: Porphyromonas gingivalis is a periodontal pathogen that resides in a complex multispecies microbial biofilm community known as dental plaque. Confocal laser scanning microscopy showed that $P$. gingivalis can assemble into communities in vitro with Streptococcus gordonii and Fusobacterium nucleatum, common constituents of dental plaque. Whole cell quantitative proteomics, along with mutant construction and analysis, were conducted to investigate how $P$. gingivalis adapts to this three species community.
\end{abstract}

Results: II56 P. gingivalis proteins were detected qualitatively during comparison of the three species model community with $P$. gingivalis incubated alone under the same conditions. Integration of spectral counting and summed signal intensity analyses of the dataset showed that 403 proteins were down-regulated and 89 proteins up-regulated. The proteomics results were inspected manually and an ontology analysis conducted using DAVID. Significant decreases were seen in proteins involved in cell shape and the formation of the cell envelope, as well as thiamine, cobalamin, and pyrimidine synthesis and DNA repair. An overall increase was seen in proteins involved in protein synthesis. HmuR, a TonB dependent outer membrane receptor, was upregulated in the community and an hmuR deficient mutant was deficient in three species community formation, but was unimpaired in its ability to form mono- or dual-species biofilms.

Conclusion: Collectively, these results indicate that $P$. gingivalis can assemble into a heterotypic community with $F$. nucleatum and $S$. gordonii, and that a community lifestyle provides physiologic support for $P$. gingivalis. Proteins such as HmuR, that are up-regulated, can be necessary for community structure. 


\section{Background}

The microbial communities that exist on oral surfaces are complex and dynamic biofilms that develop through temporally distinct patterns of microbial colonization $[1,2]$. For example, initial colonizers of the salivary pellicle on the coronal tooth surface are principally commensal oral streptococci such as $S$. gordonii and related species. Establishment of these organisms facilitates the subsequent colonization of additional gram-positives along with gramnegatives such as Fusobacterium nucleatum. As the biofilm extends below the gum line and becomes subgingival plaque, further maturation is characterized by the colonization of more pathogenic gram-negative anaerobes including Porphyromonas gingivalis [2-4]. While organisms such as $P$. gingivalis are considered responsible for destruction of periodontal tissues, pathogenicity is only expressed in the context of mixed microbial communities. Periodontal diseases, therefore, are essentially microbial community diseases, and the interactions among the constituents of these communities and between the communities and host cells and tissues, are of fundamental importance for determining the health or disease status of the periodontium.

Oral biofilm developmental pathways are driven by coadhesive, signaling and metabolic interactions among the participating organisms. Pioneer bacteria provide a substratum and appropriate metabolic support for succeeding organisms. Complex consortia then accumulate through recognition and communication systems. These interbacterial signaling processes can be based on cell-cell contact, short range soluble mediators, AI-2, or nutritional stimuli $[2,5-8]$. In general, bacterial adaptation to the community lifestyle is accompanied by distinct patterns of gene and protein expression $[9,10]$. In $S$. gordonii for example, arginine biosynthesis genes are regulated in communities with Actinomyces naeslundii which enables aerobic growth when exogenous arginine is limited [11]. Over 30 genes are differentially regulated in $P$. gingivalis following community formation with $S$. gordonii but not with $S$. mutans [12], whereas in monospecies $P$. gingivalis biofilm communities there are changes in abundance of over 80 envelope proteins [13].

While over 700 species or phylotypes of bacteria can be recovered from the oral cavity, in any one individual there are closer to 200 species [14] and the diversity of bacteria assembled in dense consortia will be further limited by nutritional and other compatibility constraints. $P$. gingivalis can accumulate into single species biofilms and mixed species consortia with $S$. gordonii and related oral streptococci [15-17]. Moreover, introduction of $P$. gingivalis into the mouths of human volunteers results in almost exclusive localization in areas of streptococcal-rich plaque [18]. Development of more complex multi-species communi- ties in aerated environments such as supragingival tooth surfaces may require oxygen scavenging by $F$. nucleatum [19]. F. nucleatum is also able to coaggregate with P. gingivalis and with oral streptococci [19-21]. Hence communities of S. gordonii, F. nucleatum and P. gingivalis are likely to be favored in vivo; however, community formation by these three organisms has not been investigated. The aim of this study was to examine the ability of S. gordonii, F. nucleatum and $P$. gingivalis to form multispecies communities in vitro, and to utilize a global proteomic approach to investigate differential protein expression in $P$. gingivalis in response to presence of these organisms.

\section{Results and discussion Assembly of P. gingivalis-F. nucleatum-S. gordonii communities in vitro}

Confocal laser scanning microscopy (CLSM) was used to investigate the ability of $P$. gingivalis to assemble into communities with $S$. gordonii and F. nucleatum. In order to mimic the temporal progression of events in vivo, S. gordonii cells were first cultured on a glass surface and this streptococcal substratum was then reacted in succession with F. nucleatum and P. gingivalis. The F. nucleatum and $P$. gingivalis cells were maintained in the absence of growth media in order to be able to detect any metabolic support being provided by the other organisms in the community. A $3 \mathrm{D}$ reconstruction of the heterotypic community is shown in Fig. 1. Both $P$. gingivalis and F. nucleatum formed discrete accumulations and could be either separate from each other or interdigitated, consistent with the concept that the later gram-negative colonizers such as $P$. gingivalis and F. nucleatum initially establish themselves on the streptococcal rich supragingival plaque $[4,18]$. The results demonstrate the mutual compatibility of these three organisms for heterotypic community development, an early step in the overall process of plaque biofilm accumulation. Participation in multispecies communities may provide a basis for synergistic interactions in virulence. For example, mixed infections of $P$. gingivalis and $F$. nucleatum are more pathogenic in animal models than either species alone [22], and F. nucleatum can enhance the ability of $P$. gingivalis to invade host cells [23].

\section{Proteome of P. gingivalis in a three species community}

To begin to investigate the mechanisms of adaptation of $P$. gingivalis to a community environment, the proteome of non-growing $P$. gingivalis cells incorporated into a community with F. nucleatum and S. gordonii was compared to the proteome of non-growing $P$. gingivalis cells alone. The expressed proteome of $P$. gingivalis in a community consisted of 1156 annotated gene products detected qualitatively. Based on spectral counting, 271 gene products showed evidence of relative abundance change at a $q$ value of 0.01: 109 proteins at higher relative abundance and 162 at lower relative abundance, using $P$. gingivalis 


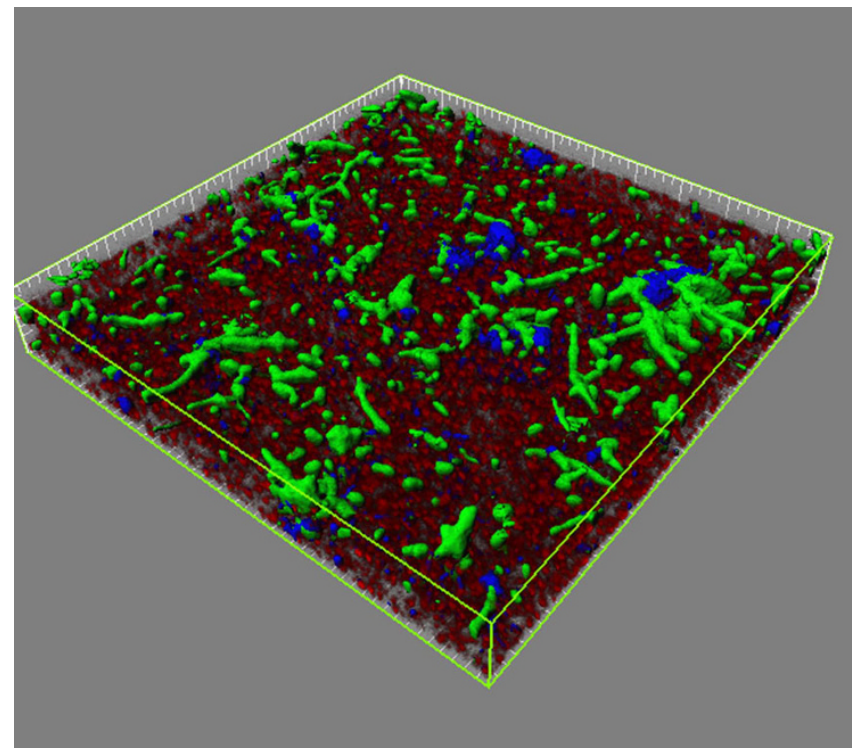

\section{Figure I}

Confocal laser scanning microscopy of $P$. gingivalis- $F$. nucleatum-S. gordonii community. S. gordonii cells (red, stained with hexidium iodide) were cultured on a glass plate. FITC-labeled F. nucleatum cells (green), followed by DAPI labeled $P$. gingivalis cells (blue), were reacted sequentially with the $S$. gordonii substratum. Bacterial accumulations were examined on a Bio-Rad Radiance 2100 confocal laser scanning microscope. A series of fluorescent optical $x-y$ sections in the z-plane to the maximum vertical extent of the accumulation were collected with Laser Sharp software. Images were digitally reconstructed with Imaris software. Image is representative of three independent experiments.

alone as a reference state. Spectral counting is a conservative measure of protein abundance change that tends to generate low FDRs [24-26] but that often suffers from high FNRs in studies of the kind described here [27]. Less conservative calculations based on intensity measurements [27] found 458 gene products with evidence of relative abundance change at a $q$-value of 0.01 : 72 proteins at higher relative abundance, and 386 proteins at lower relative abundance. Spectral counting and protein intensity measurements were examined for common trends. Trends tended to be consistent across both biological replicates, but the magnitudes of the abundance ratios showed significant scatter, similar to most published expression data at either the mRNA or protein level [27]. In most cases the abundance ratio trends were the same, using both quantitation methods, although not necessarily significantly so. In only eight cases were the spectral counting trend and summed intensity trend significantly in opposite directions for the same protein (PGN 0329, $0501,1094,1341,1637,1733,2065)$. The integrated relative abundance trends found 403 gene products with evidence of lower relative abundance change and 89 at higher relative abundance. For purposes of examining the totals for combined trends, if an abundance change was called as significant (red or green in Additional file 1: Table ST1) in one measurement, it was considered significant for the above combined totals only if the ratio of the other measurement showed the same direction of abundance change, with a $\log _{2}$ ratio of \pm 0.1 or greater regardless of the $q$-value in the second measurement. The experimental data for differential protein abundance are shown in Fig. 2 as a pseudo M/A plot $[28,29]$ with a LOWESS curve fit [30]. The same data are plotted in Fig. 3 as open reading frames according to PGN numbers from the ATCC 33277 genome annotation [31]. A complete listing of all proteins, their abundance ratios relative to $P$. gingivalis controls incubated alone under the same conditions as determined by spectral counting and summed signal

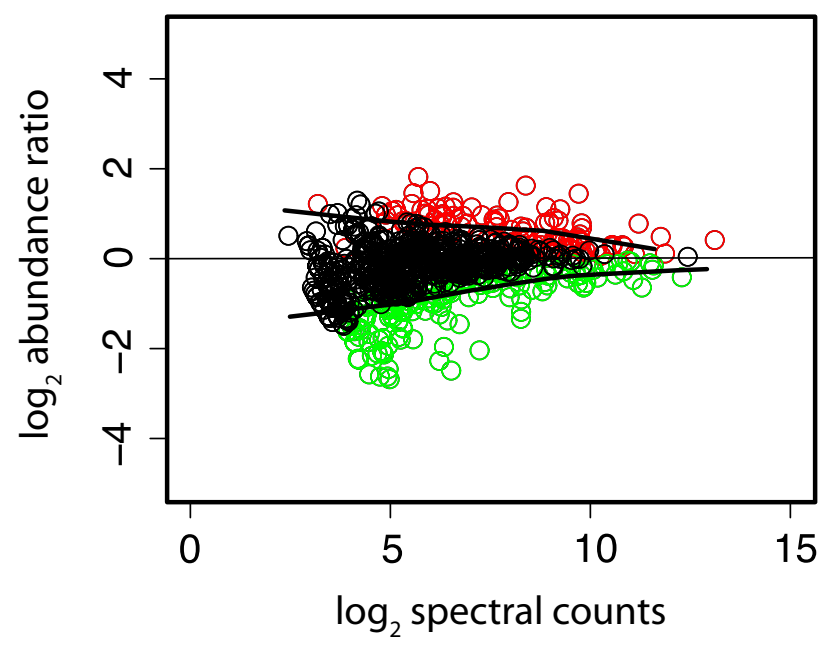

Figure 2

Pseudo $M$ versus A plot [28,29] of the average protein abundance ratios over all replicates for the $P$. gingivalis-F. nucleatum-S. gordonii/P. gingivalis comparison versus total abundance as estimated by spectral counting. Color codes: red, $P$. gingivalis protein is overexpressed in the $P$. gingivalis- $F$. nucleatum- $S$. gordonii community relative to $P$. gingivalis alone; green, $P$. gingivalis protein is under-expressed in the community relative to $P$. gingivalis alone; black, no significant abundance change. Solid black lines represent a LOWESS curve fit [30] to the biological replicates of $P$. gingivalis alone, and represent the upper and lower boundaries of the experimentally observed error regions or null distributions associated with the relative abundance ratio calculations. Proteins coded as either red or green were determined to be significantly changed at the $q$-value [24] cut-off value of 0.01 . Thus, the $G$-test predictions [56] were in good agreement with the curve fitting procedure. Details regarding hypothesis testing procedures can be found in Methods and in the explanatory notes to the data tables [see Additional File I]. 


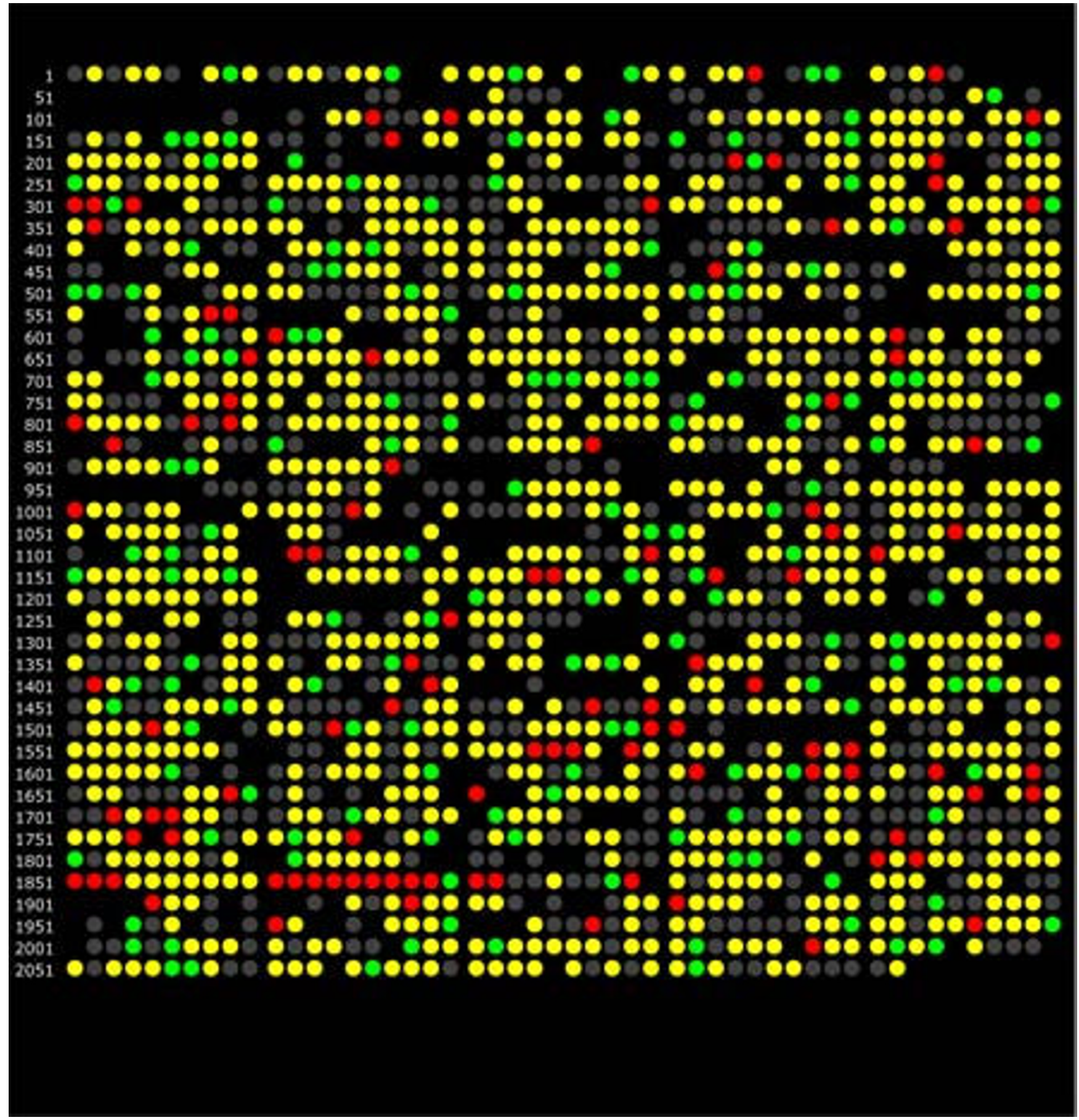

Figure 3

Genomic representation of the $P$. gingivalis proteome, showing changes in relative abundance for the $P$. gingivalis-F. nucleatum-S. gordonii/P. gingivalis comparison by spectral counting. Each dot represents a PGN ORF number in the order followed by the ATCC 33277 strain annotation. Color codes: red, over-expression in the P. gingivalis-F. nucleatum-S. gordonii community relative to $P$. gingivalis alone; green, under-expression in the community relative to $P$. gingivalis alone; yellow, protein was detected qualitatively, but did not change in abundance; gray, proteins that were qualitative non-detects; gaps indicate ORFs that were not common to both the ATCC 33277 and W83 annotations according to a master cross-reference compiled by LANL (G. Xie, personal communication). 
intensity $[27,32,33]$, and $q$-values, are given in Additional file 1: Table ST1. Qualitative identifications for proteins secreted by $P$. gingivalis in the 3 -species community but not by $P$. gingivalis alone are given Additional file 1: Table ST2. Additional file 1: Figs. SF1, SF2, SF3, SF4, SF5 and SF6 and explanatory notes provide more detailed technical information regarding reproducibility of the biological replicates and the adequacy of sampling depth. To assess global sampling depth, average spectral counts were calculated by summing all spectral count numbers for all $P$. gingivalis proteins in the FileMaker script output described under Methods and dividing by the total number of $P$. gingivalis proteins in that file. The average redundant spectral count number for peptides unique to a given ORF for $P$. gingivalis alone was 80 , for $P$. gingivalis in the community it was 64 . The lower number of counts observed for $P$. gingivalis proteins in the community is consistent with the added sampling demands placed on the analytical system by sequence overlaps in the proteomes of all three microbes and thus the smaller number of unique proteolytic fragments predicted. More discussion of this topic is given in the explanatory notes [see Additional File 1]. Spectral count values for individual proteins are given in data Additional file 1: Table ST1. Details regarding access to mass spectrometry data for individual peptides and their SEQUEST database searching scores [34], p-values and $q$-values are given in the notes to the data tables [see Additional File 1].

\section{Proteins and functions differentially regulated by $\mathbf{P}$. gingivalis in a community \\ Cell envelope and cell structure}

In bacterial communities significant surface-surface contact occurs both within and among accumulations of the constituent species, as was also observed in the P. gingivalis-F. nucleatum-S. gordonii consortia. Regulation of outer membrane constituents of $P$. gingivalis would thus be predicted in the context of a community and this was borne out by the proteomic results. Overall, 84 proteins annotated as involved in the cell envelope were detected, and 40 of these showed reduced abundance in the three species community, indicating an extensive change to the cell envelope. Only four proteins showed increased abundance, two OmpH proteins (PGN0300, PGN0301) and two lipoproteins (PGN1037, PGN1998). MreB (PGN0234), a bacterial actin homologue that plays a role in determining cell shape, showed almost a 2-fold decrease in community derived $P$. gingivalis. Expression of MreB has been found to decrease under stress or during stationary phase in Vibrio paraheamolyticus [35]. However, stress-related proteins were generally reduced in $P$. gingivalis cells in the community (see below) so stress is an unlikely explanation for the change in MreB. Rather, the decrease in MreB abundance may be due to the P. gingivalis cells entering a state resembling stationary phase or responding in a previously unseen way to the formation of the three species community.

\section{Protein synthesis}

Extensive changes were observed in ribosomal proteins and in translation elongation and initiation proteins. While overall more proteins showed reduced abundance in the three species community, the changes to the translational machinery were almost exclusively increases in abundance. Of 49 ribosomal proteins detected, 27 showed increased abundance, while only one showed decreased abundance. Of nine translation elongation and initiation proteins detected, none showed significant abundance decreases but five showed increased abundance (EfG (PGN1870), putative EfG (PGN1014), EfTs (PGN1587), EfTu (PGN1578), and If2 (PGN0255)). This represents not only a substantial portion of the translational machinery but also a large portion, $36 \%$, of the proteins showing increased abundance. It is well known that ribosomal content is generally proportional to growth rate [36]; however, given that the cells were not in culture medium during the assay, rapid growth is an unlikely explanation for these results. The increased ribosomal content presumably indicates increased translation, consistent with the community providing physiologic support to $P$. gingivalis and allowing higher levels of protein synthesis.

\section{Vitamin synthesis}

Pathways for synthesizing several vitamins showed reduced protein abundance in the three species community. Most of the proteins involved in thiamine diphosphate (vitamin B1) biosynthesis were downregulated (Fig. 4 ). Thiamine is a cofactor for the 2-oxoglutarate dehydrogenase complex that converts 2-oxoglutarate to succinylCoA and for the transketolase reactions of the anaerobic pentose phosphate pathway [37]. However, transketolase (PGN1689, Tkt) showed no abundance change while of the three components of the 2-oxoglutarate dehydrogenase complex (PGN1755, KorB) only the beta subunit showed an abundance increase.

Only incomplete pathways have been identified for many of the other vitamin biosynthesis activities in $P$. gingivalis. However, cobalamin (vitamin B12) synthesis [38] can be predicted to be decreased in the community, with five (PGN0010, CobC; PGN0316, CbiG; PGN0317, CobL; PGN0318, CobH/CbiC; PGN0735, CobU) of the seven identified proteins having statistically significant reductions. Less complete population of pathways was observed for pyridoxal phosphate (vitamin B6) and biotin synthesis. Only two of the four detected proteins for vitamin B6 synthesis showed reduced abundance (PGN1359, PdxB and PGN2055, PdxA). For biotin synthesis, three of the six detected proteins showed reduced abundance 


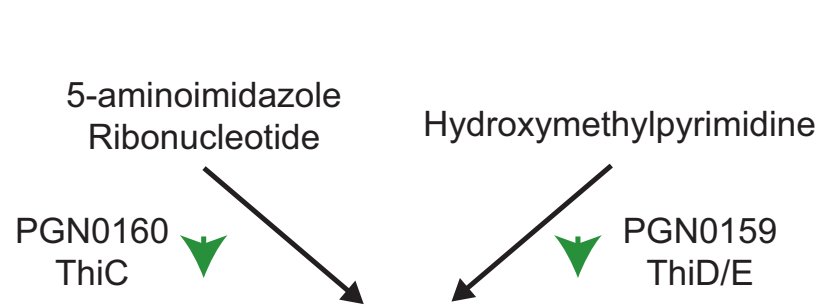

Hydroxymethylpyrimidine Phosphate

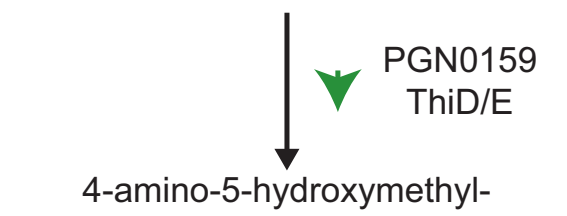

2-methylpyrimidine- pyrophosphate
D-Glyceraldehyde-

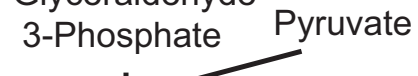

5-Phosphate

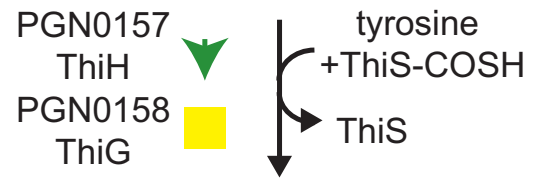

4-Methyl-5-(B-hydroxyethyl)-

thiazole Phosphate

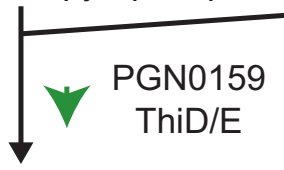

Thiamine Phosphate

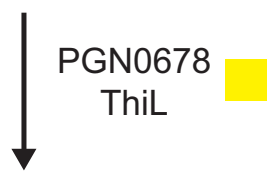

PGN1516

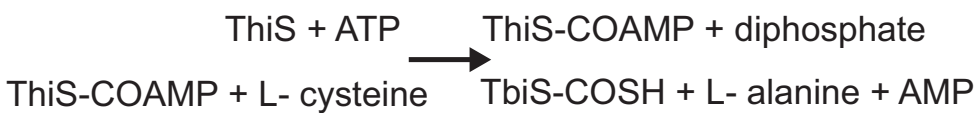

\section{Thiamine Diphosphate}

\section{Figure 4}

Thiamine biosynthetic pathway, showing protein abundance changes for the $P$. gingivalis-F. nucleatum-S. gordonii/P. gingivalis comparison. Proteins catalyzing each step in the pathway are shown by their P. gingivalis ATCC 33277 gene designation (PGN number) and protein name, where applicable. Green downward arrows indicate decreased abundance in the three species community. Yellow squares indicate no statistically significant abundance change. Empty squares indicate that the protein was not detected in the proteomic analysis. Thiamine diphosphate is shown in bold.

(PGN0133, BioA; PGN1721, BioF; PGN1997, BioD). None of the vitamin/cofactor synthesis pathways showed any indication of increased protein levels in the three species community.

The decrease in several vitamin/cofactor pathways could be due to a decreased utilization of those cofactors. However, in the case of thiamine, the proteins that utilize this cofactor showed no decrease, and a possible increase in abundance, implying that demand for vitamin B1 was unchanged. A more likely explanation for the reduced cofactor pathways is therefore nutrient transfer. Either one or both of the other organisms in the three species community could be providing $P$. gingivalis with cofactors, allowing reduced cofactor synthesis without reducing expression of the cofactor dependent pathways. Nutritional cross-feeding among members of oral biofilms is well established [5], and indeed $P$. gingivalis has been found to utilize succinate produced by T. denticola [39].

\section{Nucleotide synthesis}

Pyrimidine biosynthesis appeared to be reduced in the three species community (Fig. 5) as many of the proteins leading to the production of finished pyrimidine nucleotides have decreased abundance. However, the proteins responsible for incorporating finished ribonucleotides into RNA show unchanged or increased abundance. As with vitamin biosynthesis this may be the result of nutrient transfer from the other organisms in the community. $P$. gingivalis can acquire nucleosides and nucleobases and it has even been suggested that they may represent an important nutrient source for $P$. gingivalis [40]. Consistent with uptake of nucleosides and their precursors, uracil permease (PGN1223) shows increased expression in the three species community.

Purine biosynthesis does not appear to be significantly effected in the three species community (Fig. 6). A few proteins showed reduced abundance, but the central biosynthesis pathway was primarily unchanged. 


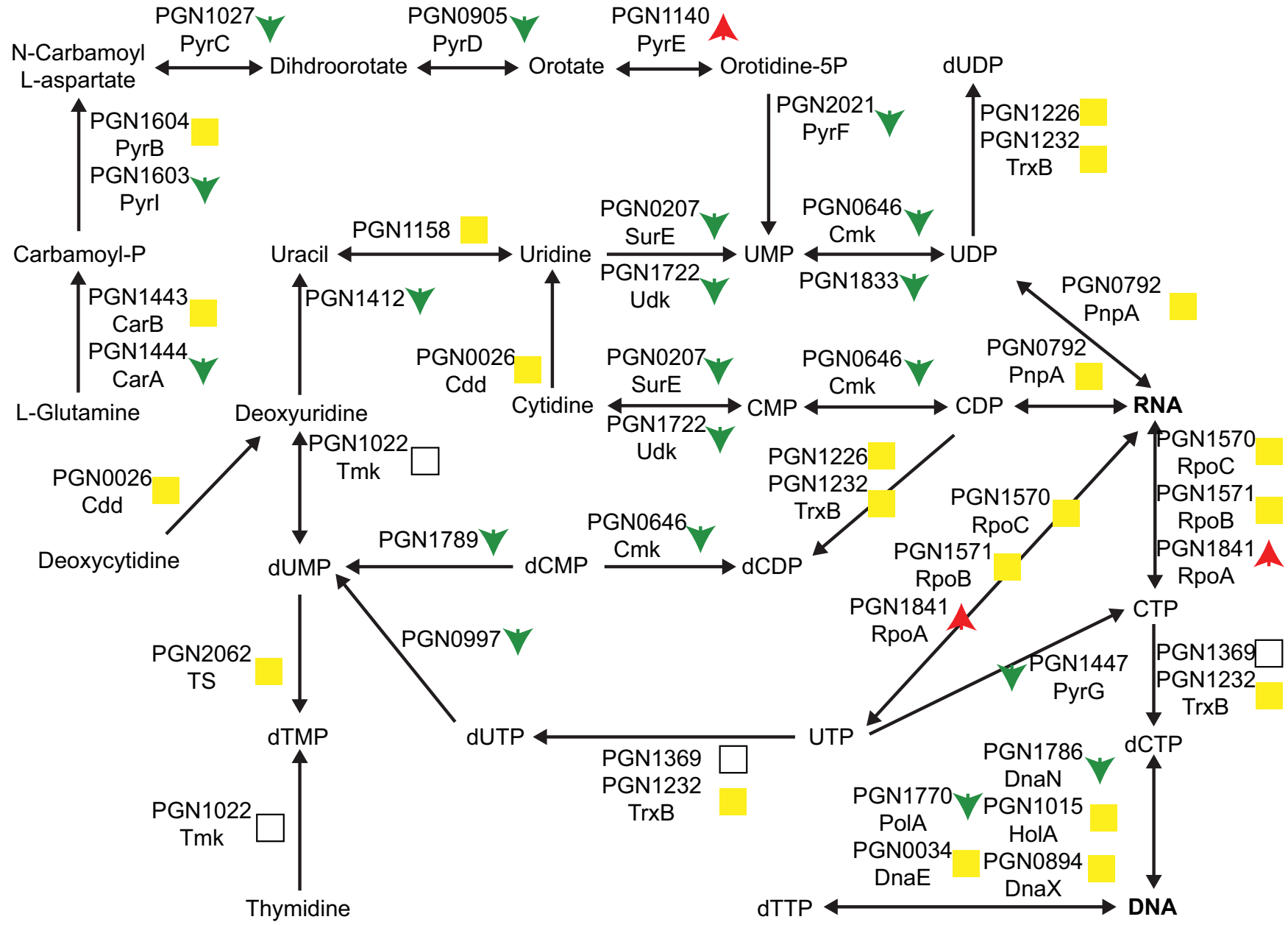

Figure 5

Pyrimidine biosynthetic pathway, showing protein abundance changes for the $P$. gingivalis-F. nucleatum-S. gordonii/P. gingivalis comparison. The protein names follow the same conventions as in Fig. 4. Green downward arrows indicate decreased abundance in the three species community. Red upward arrows indicate increased abundance. Yellow squares indicate no statistically significant abundance change. Empty squares indicate that the protein was not detected in the proteomic analysis. RNA and DNA are shown in bold.

\section{Stress proteins}

The ability of the community to provide physiologic support to constituent species might result in $P$. gingivalis experiencing lower levels of environmental stress than occurs in monoculture. Consistent with this concept, community derived $P$. gingivalis showed a significant reduction in abundance of DNA repair proteins (PGN0333, RadA; PGN0342, Ung; PGN0367, Xth; PGN1168, MutS; PGN1316, UvrA; PGN1388, LigA; PGN1567, RecF; PGN1585, UvrB; PGN1712, Nth; PGN1714, Mfd; PGN1771, Pol1). DNA repair genes are generally induced in the presence of damaged DNA [41], and lower abundance of DNA repair proteins is consistent with the monoculture experiencing more DNA damage than $P$. gingivalis in the three species community where the presence of the partner organisms provides protection against DNA damage.

Only two stress proteins showed increased abundance, and then only 30\% increases, the molecular chaperone DnaK (PGN1208) and a PhoH family protein possibly involved in oxidation protection (PGN0090).

\section{Role of the differentially regulated $\mathbf{P}$. gingivalis protein HmuR}

To begin to test the functional relevance of proteins identified as differentially regulated in the three species community, we undertook a mutational analysis. For this 


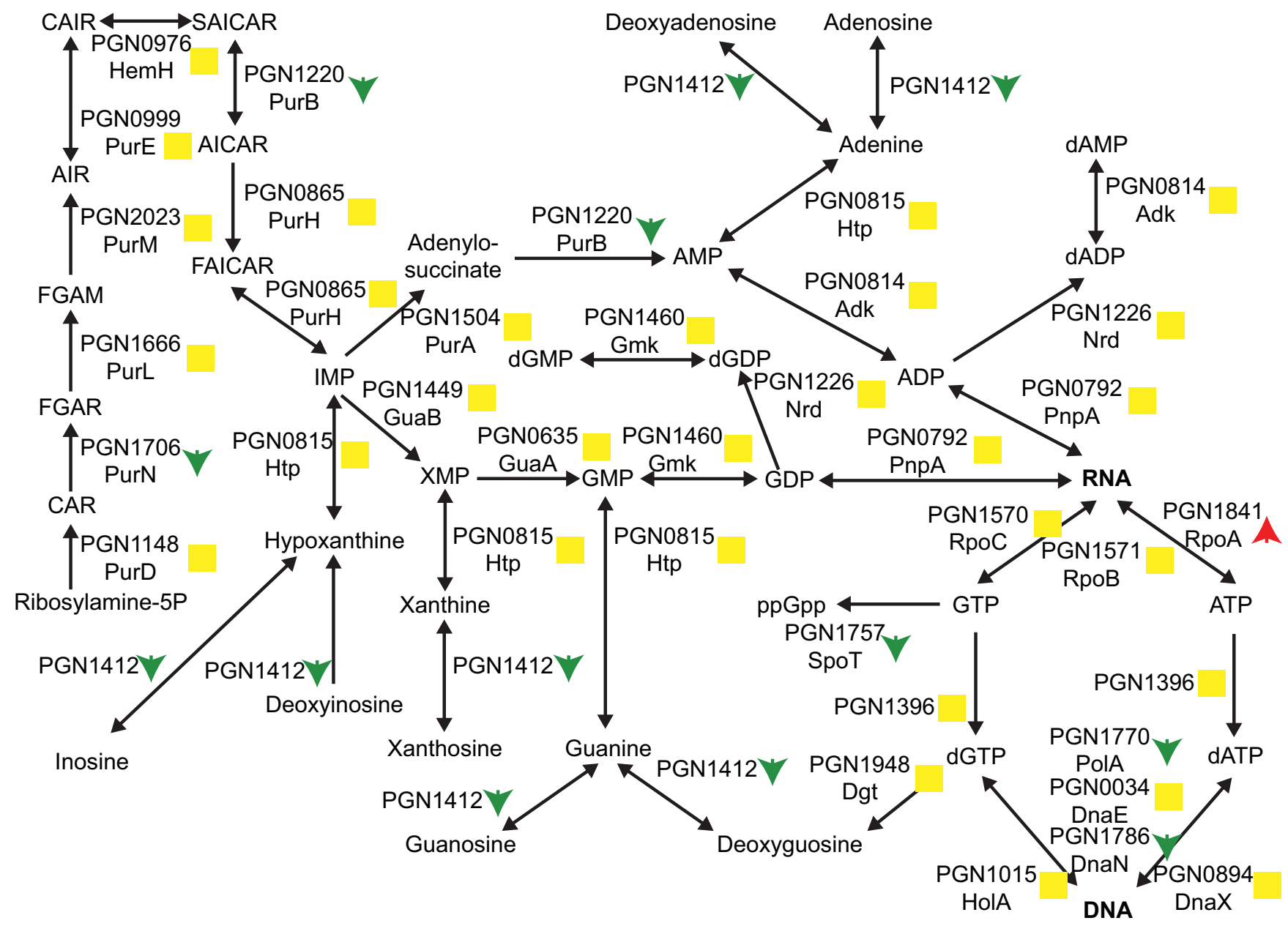

Figure 6

Purine biosynthetic pathway, showing protein abundance changes for the $P$. gingivalis-F. nucleatum-S. gordonii/P. gingivalis comparison. The protein names and arrows/squares follow the same conventions as in Fig. 5. RNA and DNA are shown in bold. GAR: 5-Phosphoribosyl glycinamide; FGAM: 5-phosphoribosyl-N-formylglycineamidine; FGAR: I-(5'-Phosphoribosyl)-N-formylglycinamide; AICAR: 5'-phosphoribosyl-4-(N-succinocarboxamide)-5-aminoimidazole; AIR: I-(5'-Phophoribosyl)-5-aminoimidazole; CAIR: 5'P-Ribosyl-4-carboxy-5-aminoimidazole; SAICAR: 5'P-Ribosyl-4-(N-succinocarboximide)-5aminoimidazole; FAICAR: I (5'-Phosphoribosyl)-5-formamido-4-imidazole carboxamide.

purpose it was important to target a protein that directly effectuates a biological function and lacks homologs in the genome. HmuR, a major hemin uptake protein, and potential adhesin [42], was selected. As shown in Fig. 7A, while wild type $P$. gingivalis cells are abundant within a $S$. gordonii-F. nucleatum-P. gingivalis community, $P$. gingivalis cells lacking HmuR are deficient in community formation. Biovolume analysis showed a $70 \%$ reduction in community formation by the HmuR mutant (Fig. 7C). Furthermore, this effect was specific for the three species community as a decrease in accumulation by the HmuR deficient mutant was not observed in monospecies biofilms, or in two species communities of $P$. gingivalis with either S. gordonii or F. nucleatum (Fig. 7B, D-G). Hence loss of HmuR, that is up-regulated by $P$. gingivalis when the organism is associated with $S$. gordonii and F. nucleatum, results in a phenotype that is restricted to three species community formation. $P$. gingivalis cells were first cultured in hemin excess, under which conditions the hmu operon is expressed at a basal level [42]. As the three species model system involves metabolically quiescent $P$. gingivalis cells in buffer, it is unlikely that the role of HmuR is related to its hemin uptake capacity. However, TonB dependent receptors can exhibit functions distinct from transport across the outer membrane. For example, in $E$. coli the TonB dependent catecholate siderophore receptor 


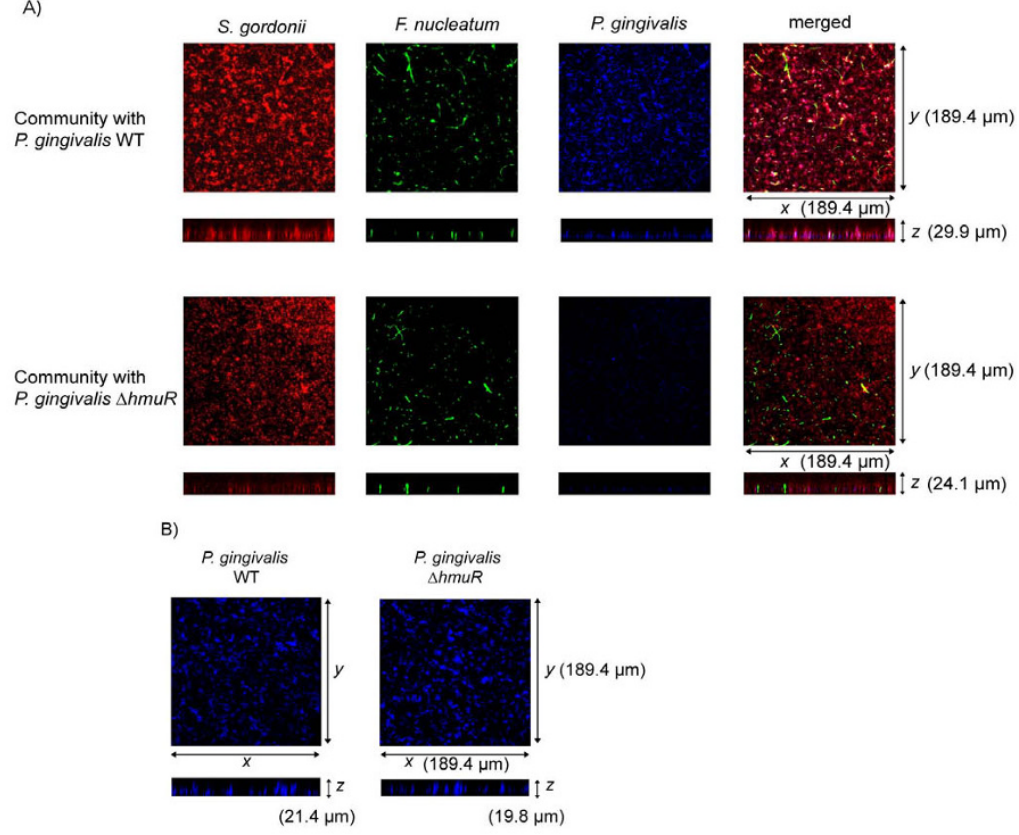

D)
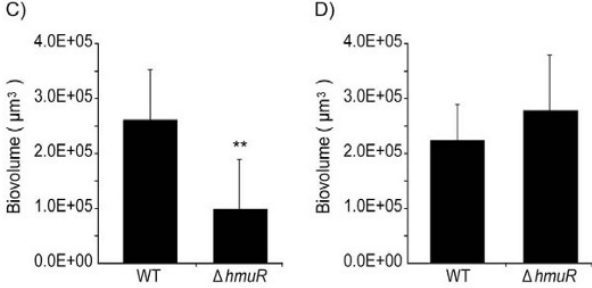

E)

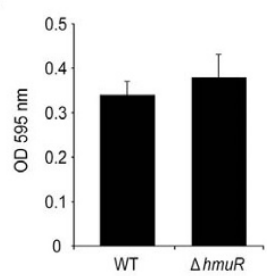

F)
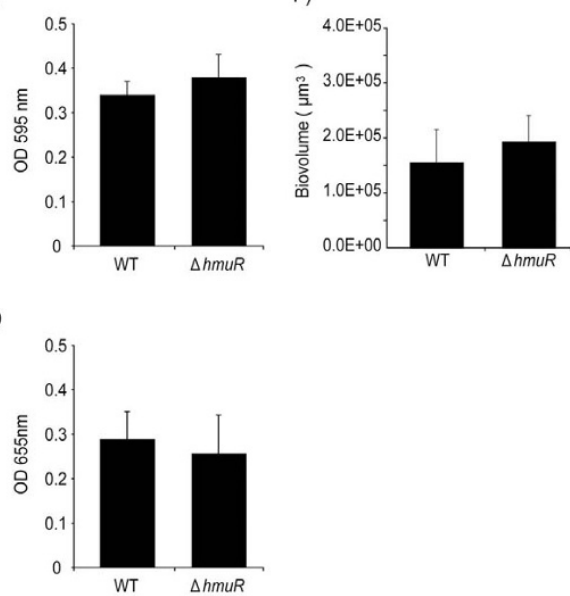

Figure 7

HmuR mutant of $P$. gingivalis is deficient in community accumulation. A) Confocal microscopy showing $x-y$ and $x-z$ projections of communities of $S$. gordonii (red), F. nucleatum (green) and P. gingivalis (blue) wild type (WT) or $\Delta h m u R$ mutant strains. Representative image from three independent experiments. B) Confocal microscopy showing $x-y$ and $x-z$ projections of single species $P$. gingivalis WT or $\Delta h m u R$ mutant accumulations. Representative image from three independent experiments. C) Biovolume analysis of $P$. gingivalis WT or $\Delta h m u R$ mutant accumulation in the $P$. gingivalis- $F$. nucleatum-S. gordonii communities shown in A. D) Biovolume analysis of $P$. gingivalis WT or $\triangle h m u R$ single species accumulations shown in $B$. E) Biomass of $P$. gingivalis WT or $\Delta h m u R$ single species accumulations measured by crystal violet staining and release. F) Biovolume analysis of $P$. gingivalis WT or $\Delta h m u R$ accumulation in two species $P$. gingivalis-S. gordonii communities. G) Biomass of $P$. gingivalis WT or $\Delta h m u R$ two species accumulation with $F$. nucleatum measured with $P$. gingivalis antibodies. ** denotes $p<0.01(n=3)$ compared to WT. 
Iha confers an adhesin function and contributes to colonization and virulence in the mouse urinary tract [43]. Hence, HmuR may have a cohesive function in community formation by $P$. gingivalis although further studies are necessary to resolve this issue.

\section{Conclusion}

Complex multi-species biofilms such as pathogenic dental plaque accumulate through a series of developmental steps involving attachment, recruitment, maturation and detachment. Choreographed patterns of gene and protein expression characterize each of these steps. In this study we developed a model of the early stages of plaque development whereby three compatible species accreted into simple communities. $P$. gingivalis increased in biomass due to attachment and recruitment, and this allowed us to catalog differential protein expression in $P$. gingivalis consequent to contact dependent interbacterial signaling and communication through short range soluble mediators. The proteomic analysis indicated that around $40 \%$ of $P$. gingivalis proteins exhibit changes in abundance in a community with $F$. nucleatum and S. gordonii, implying extensive interactions among the organisms. The proteomic results were consistent with the formation of a favorable environment in a $P$. gingivalis- $F$. nucleatum-S. gordonii community, wherein $P$. gingivalis showed evidence of increased protein synthesis and decreased stress. Moreover, nutrient transfer may occur among the constituents of the community. As evidenced by HmuR, these proteins may have a functional role in the development of multispecies communities and ultimately shape the pathogenic potential of plaque.

\section{Methods}

\section{Bacteria and culture conditions}

Fusobacterium nucleatum subsp. nucleatum ATCC 25586 and Porphyromonas gingivalis ATCC 33277 were grown anaerobically $\left(85 \% \mathrm{~N}_{2}, 10 \% \mathrm{H}_{2}, 5 \% \mathrm{CO}_{2}\right)$ at $37^{\circ} \mathrm{C}$ in trypticase soy broth supplemented with $1 \mathrm{mg} / \mathrm{ml}$ yeast extract, $1 \mu \mathrm{g} / \mathrm{ml}$ menadione and $5 \mu \mathrm{g} / \mathrm{ml}$ hemin (TSB). $S$. gordonii DL1 was grown anaerobically at $37^{\circ} \mathrm{C}$ in ToddHewitt broth (THB).

\section{Chemicals}

HPLC grade acetonitrile was from Burdick \& Jackson (Muskegon, MI, USA); high purity acetic acid (99.99\%) and ammonium acetate (99.99\%), from Aldrich (Milwaukee, WI, USA). High purity water was generated with a NANOpure UV system (Barnstead, Dubuque, IA, USA).

\section{Proteomics of model bacterial communities}

High density bacterial communities were generated by the method of Merritt et al. [44]. Bacteria were cultured to mid-log phase, harvested by centrifugation and resuspended in pre-reduced PBS (rPBS). $1 \times 10^{9}$ cells of $P$. gin- givalis were mixed with an equal number of $S$. gordonii and F. nucleatum as a combination of the three species. P. gingivalis cells alone were also used as a control. Two independent biological replicates from separate experiments comprised of at least two technical replicates were analyzed. Bacteria were centrifuged at $3000 \mathrm{~g}$ for $5 \mathrm{~min}$, and pellets were held in $1 \mathrm{ml}$ pre-reduced $\mathrm{PBS}$ in an anaerobic chamber at $37^{\circ} \mathrm{C}$ for $18 \mathrm{~h}$. The bacterial cells remain viable under these conditions, as determined by both colony counts and live/dead fluorescent staining. Supernatant and bacterial cells were separated and processed separately. Bacterial cells were lysed with ice cold sterile distilled water and proteins were digested with trypsin as previously described for $P$. gingivalis [33], then fractionated on a $2.0 \mathrm{~mm} \times 150 \mathrm{~mm}$ YMC polymer C18 column. There were five pre-fractions collected for each cellular sample, with a final volume of $50 \mu \mathrm{l}$ for each fraction. The 2D capillary HPLC/MS/MS analyses $[32,45,46]$ were conducted using an in-house fabricated semi-automated system, consisting of a Thermo LTQ mass spectrometer (Thermo Fisher Corp. San Jose, CA, USA), a Magic 2002 HPLC (Michrom BioResouces, Inc., Auburn, CA, USA), a Pump 11 Plus syringe pump (Harvard Apparatus, Inc., Holliston, MA, USA), an Alcott 718 autosampler (Alcott Chromatography, Inc., Norcross, GA, USA) and a microelectrospray interface built in-house. About $2 \mu \mathrm{l}$ of sample solution was loaded into a $75 \mu \mathrm{m}$ i.d. $\times 360 \mu \mathrm{m}$ o.d. capillary column packed with $11 \mathrm{~cm}$ of AQUA C18 $(5 \mu \mathrm{m}$, Phenomenex, Torrance, CA, USA) and $4 \mathrm{~cm}$ of polysulfoethyl aspartamide SCX (strong cation exchange) resin (PSEA, $5 \mu \mathrm{m}$, Michrom BioResouces, Inc.). The peptides were eluted with a seven step salt gradient $(0,10,25,50$, 100,250 and $500 \mathrm{mM}$ ammonium acetate) followed by an acetonitrile gradient elution (Solvent A: 99.5\% water, $0.5 \%$ acetic acid. Solvent B: $99.5 \%$ acetonitrile, $0.5 \%$ acetic acid), 5\% B hold $13 \mathrm{~min}, 5-16 \% \mathrm{~B}$ in $1 \mathrm{~min}$, hold 6 min, $16-45 \%$ B in $45 \mathrm{~min}, 40-80 \%$ B in 1 min, hold 9 $\min , 80-5 \%$ B in $5 \mathrm{~min}$, then hold $10 \mathrm{~min}$. For the secreted proteins in the supernatant no pre-fractionation or SCX was performed, and $4 \mu \mathrm{l}$ of digested sample was loaded into a $75 \mu \mathrm{m}$ i.d. $\times 360 \mu \mathrm{m}$ o.d. column packed with $11 \mathrm{~cm}$ AQUA C18 for a single dimension of capillary HPLC/tandem MS analysis. After 20 min of flushing with $5 \%$ acetonitrile, peptides were eluted by an acetonitrile gradient (5-12\% B in $1 \mathrm{~min}$, hold $9 \mathrm{~min}, 12-40 \% \mathrm{~B}$ in 50 min, $40-80 \%$ B in $1 \mathrm{~min}$, hold $10 \mathrm{~min}, 80-5 \% \mathrm{~B}$ in $5 \mathrm{~min}$, hold $14 \mathrm{~min}$ ). The $\mathrm{MS}^{1}$ scan range for all samples was $400-2000 \mathrm{~m} / z$. Each $\mathrm{MS}^{1}$ scan was followed by $10 \mathrm{MS}^{2}$ scans in a data dependent manner for the 10 most intense ions in the MS ${ }^{1}$ scan. Default parameters under Xcalibur 1.4 data acquisition software (Thermo Fisher) were used, with the exception of an isolation width of $3.0 \mathrm{~m} / \mathrm{z}$ units and a normalized collision energy of $40 \%$. 


\section{Data processing and protein identification}

Raw data were searched by SEQUEST [34] against a FASTA protein ORF database consisting of the Ver. 3.1 curation of $P$. gingivalis W83 (2006, TIGR-CMR [47]), S. gordonii Challis NCTC7868 (2007, TIGR-CMR [48], F. nucleatum ATCC 25586 (2002, TIGR-CMR [49]), bovine (2005, UC Santa Cruz), nrdb human subset (NCBI, as provided with Thermo Bioworks ver. 3.3) and the MGC (Mammalian Gene collection, 2004 curation, NIH-NCI [50]) concatenated with the reversed sequences. After data processing, the genome sequence for strain 33277 became available [31] and the data were subsequently cross-referenced to PGN numbers from the 33277 specific FASTA database provided by LANL (personal communication with G. Xie). Although Naito et al. [31] reported extensive genome re-arrangements between W83 and ATCC 33277, the actual protein amino acid sequences are sufficiently similar across the proteome that the use of a database based on W83 was not expected to greatly impact the analysis. Our proteomic methods are not sensitive to genome re-arrangements, only to changes in amino acid sequence for a given protein. The reversed sequences were used for purposes of calculating a peptide level qualitative FDR using the published method [51,52]. The SEQUEST peptide level search results were filtered and grouped by protein using DTASelect [53], then input into a FileMaker script developed in-house [32,33] for further processing. The DTASelect Ver. 1.9 filter parameters were: peptides were fully tryptic; $\Delta \mathrm{Cn} / \mathrm{Xcorr}$ values for different peptide charge states were $0.08 / 1.9$ for $+1,0.08 / 2.0$ for +2 , and $0.08 / 3.3$ for +3 ; all spectra detected for each sequence were retained $(\mathrm{t}=0)$. Only peptides that were unique to a given ORF were used in the calculations, ignoring tryptic fragments that were common to more than one ORF or more than one organism, or both. In practice this had the consequence of reducing our sampling depth from what we have achieved with single organism studies [27,32,33], because the gene sequence overlap among the three organisms is significant. A bioinformatic analysis (data not shown) of inferred protein sequence overlaps between $P$. gingivalis and $S$. gordonii or F. nucleatum suggested the reduction in the number of predicted tryptic fragments unique to $P$. gingivalis would not be sufficient to impact the analysis of more than a small number of proteins. The qualitative peptide level FDR was controlled to approximately $5 \%$ for all conditions by selecting a minimum non-redundant spectral count cut-off number appropriate to the complexity of each condition, $P$. gingivalis alone or the $P$. gingivalis-F. nucleatum-S. gordonii community.

\section{Protein abundance ratio calculations}

Protein relative abundances were estimated on the basis of summed intensity or spectral count values [27,32,33] for proteins meeting the requirements for qualitative identification described above. Summed intensity refers to the summation of all processed parent ion (peptide) intensity measurements $\left(\mathrm{MS}^{1}\right)$ for which a confirming CID spectrum $\left(\mathrm{MS}^{2}\right)$ was acquired according to the DTASelect filter files. For spectral counts, the redundant numbers of peptides uniquely associated with each ORF were taken from the DTAselect filter table $(\mathrm{t}=0)$. Spectral counting is a frequency measurement that has been demonstrated in the literature to correlate with protein abundance [54]. These two ways of estimating protein relative abundance, that avoid the need for stable isotope labeling, have been discussed in a recent review [27] with specific reference to microbial systems. To calculate protein abundance ratios, a normalization scheme was applied such that the total spectral counts or total intensities for all $P$. gingivalis proteins in each condition were set equal for each comparison. This normalization also had the effect of zero centering the $\log _{2}$ transformed relative abundance ratios, see Fig. 2 (and also the frequency histograms in Additional file 1: Figs. SF5 and SF6). The normalized data for each abundance ratio comparison was tested for significance using either a global G-test or a global paired $t$-test for each condition, the details of which have been published for this type of proteomics data in which all biological replicates are compared against each other $[55,56]$, and are also described in the explanatory notes [see Additional File 1]. Both of these testing procedures weigh deviation from the null hypothesis of zero abundance change and random scatter in the data to derive a probability or $p$-value that the observed change is a random event, i.e. that the null hypothesis of no abundance change is true. Each hypothesis test generated a p-value that in turn was used to generate a $q$-value as described [24,32], using the R package QVALUE [26]. The $q$-value in this context is a measure of quantitative FDR [25] that contains a correction for multiple hypothesis testing. A $q$ cut-off value of 0.01 was used for all ratios reported in Additional file 1: Table ST1. All statistical calculations were done in $\mathrm{R}$ (Ver. 2.5.0), using source code that has been published $[32,33,55]$. Only proteins with data consisting of confirmed high scoring $\mathrm{MS}^{2}$ mass spectra (high scoring qualitative database matches as described above) present in both the numerator and denominator of the abundance ratio comparison were listed as significantly changed in Additional file 1. Certain proteins listed in the tables with $q$-values $=0.01$ are still coded yellow for no significant abundance change due to missing data in either the numerator or the denominator.

\section{Ontology analysis}

An overall list of detected proteins as well as lists of proteins that showed increased or decreased levels in the three species community were prepared using Entrez gene 
identifiers. Ontology analyses were then conducted using the DAVID [57] functional annotation clustering feature with the default databases. Both increased and decreased protein level lists were analyzed using the overall list of detected proteins as the background. Potentially interesting clusters identified by DAVID were then examined manually.

\section{Construction of $\mathbf{P}$. gingivalis HmuR mutant}

A mutation in the $h m u R$ gene was generated using ligation-independent cloning of PCR mediated mutagenesis (LIC-PCR) [58]. A 2.1-kb ermF-ermAM cassette was introduced into the $h m u R$ gene by three steps of PCR to yield a $h m u R-e r m-h m u R$ DNA fragment as described previously [59]. The fragment was then introduced into $P$. gingivalis 33277 by electroporation. The hmuR deficient mutant $(\Delta h m u R)$ was generated via a double crossover event that replaces $h m u R$ with the hmuR-erm-hmuR DNA fragment in the 33277 chromosome. The mutants were selected on TSB plates containing erythromycin $(5 \mu \mathrm{g} / \mathrm{ml})$, and the mutation was confirmed by PCR analysis. Growth rates of mutant and parent strains were equivalent.

\section{Quantitative community development assays}

i) Crystal violet assay. Homotypic community formation by $P$. gingivalis was quantified by a microtiter plate assay [60], as adapted for $P$. gingivalis [61]. Parental and mutant strains in early log phase $\left(2 \times 10^{8}\right.$ cells $)$ were incubated at $37^{\circ} \mathrm{C}$ anaerobically for $24 \mathrm{~h}$. Wells were washed, stained with $1 \%$ crystal violet and destained with 95\% ethanol. Absorbance at $595 \mathrm{~nm}$ was determined in a Benchmark microplate reader. ii) ELISA. F. nucleatum was incubated at $37^{\circ} \mathrm{C}$ anaerobically for $36 \mathrm{~h}$ in microtiter plate wells. After washing, parental and mutant $P$. gingivalis strains $\left(2 \times 10^{6}\right.$ cells) were incubated with the fusobacterial biofilm at $37^{\circ} \mathrm{C}$ anaerobically for $24 \mathrm{~h}$. P. gingivalis accumulation was detected with antibodies to whole cells $(1: 10,000)$ followed by peroxidase-conjugated secondary antibody $(1: 3,000)$, each for $1 \mathrm{~h}$ at $37^{\circ} \mathrm{C}$. Antigen-antibody binding was determined by a colorimetric reaction using the 3,3',5,5'-tetramethylbenzidine (TMB) liquid substrate, and absorbance at $655 \mathrm{~nm}$. P. gingivalis antibody binding to the fusobacterial biofilm alone was subtracted as background. iii) Confocal microscopy assay. A. Single species. $P$. gingivalis was stained with 4',6-diamidino-2-phenylindole $\left(50 \mu \mathrm{g} \mathrm{ml}^{-1}\right)$ and $2 \times 10^{6}$ cells in rPBS incubated anaerobically at $37^{\circ} \mathrm{C}$ for $16 \mathrm{~h}$ with rocking in individual chambers of the CultureWell coverglass system (Grace Bio Labs). Chambers were washed three times in rPBS. B. Dual species. Heterotypic $P$. gingivalis-S. gordonii communities were generated as described previously [15]. S. gordonii cells were labeled with hexidium iodide $\left(15 \mu \mathrm{g} \mathrm{ml}^{-1}\right)$, then cultured anaerobically at $37^{\circ} \mathrm{C}$ for $16 \mathrm{~h}$ with rocking in CultureWell chambers. P. gingivalis was stained with 5- (and-6)-carboxyfluorescein, succinimidyl ester $(10 \mu \mathrm{g} \mathrm{ml}$ $\left.{ }^{1}\right)$, and $2 \times 10^{6}$ cells in rPBS were reacted with the surface attached $S$. gordonii for $24 \mathrm{~h}$ anaerobically at $37^{\circ} \mathrm{C}$ with rocking. C) Three species. Surface attached hexidium iodide-stained $S$. gordonii were generated as above. Fluorescein stained $F$. nucleatum $\left(2 \times 10^{6}\right.$ cells in rPBS) reacted with $S$. gordonii for $24 \mathrm{~h}$ anaerobically at $37^{\circ} \mathrm{C}$ with rocking. The coverglass was then washed with rPBS to remove non-attached bacteria. $P$. gingivalis was stained with 4 ',6diamidino-2-phenylindole ( $\left.50 \mathrm{\mu g} \mathrm{ml}^{-1}\right)$ and $2 \times 10^{6}$ cells in rPBS were added and further incubated for $24 \mathrm{~h}$ anaerobically at $37^{\circ} \mathrm{C}$ with rocking. Communities were observed on a Bio-Rad Radiance 2100 confocal laser scanning microscope (Blue Diode/Ar/HeNe) system with an Nicon ECLIPSE TE300 inverted light microscope and $40 \times$ objective using reflected laser light of combined 405, 488 and $543 \mathrm{~nm}$ wavelengths where appropriate. A series of fluorescent optical $x-y$ sections were collected to create digitally reconstructed images (z-projection of $x-y$ sections) of the communities with Image J V1.34s (National Institutes of Health) or Laser Sharp software (Bio-Rad). Z stacks of the x-y sections of CLSM were converted to composite images with "Iso Surface" functions of the "Surpass" option on Imaris 5.0.1 (Bitplane AG; Zurich, Switzerland) software. Iso Surface images of $P$. gingivalis were created at threshold of 20 and smoothed with Gaussian Filter function at 0.5 width, and $P$. gingivalis biovolume was calculated.

Biofilm assays were repeated independently three times with each strain in triplicate. Crystal violet results were compared by $t$-tests. Biovolume calculations were compared with a $t$-test using the SPSS statistics software.

\section{Abbreviations}

ATCC: American Type Culture Collection; DAVID: Database for Annotation, Visualization and Integrated Discovery; FDR: false discovery rate; FNR: false negative rate; LANL: Los Alamos National Laboratory; LOWESS: Locally weighted scatterplot smoothing; MS: Mass spectrometry; $\mathrm{MS}^{1}$ : First stage of tandem mass spectrometry; $\mathrm{MS}^{2}$ : Second stage of tandem mass spectrometry; SCX: strong cation exchange; TIGR-CMR: The Institute for Genomic Research Comprehensive Microbial Resource, now part of the J. Craig Venter Institute.

\section{Authors' contributions}

MK carried out the community construction and analysis by confocal microscopy; ELH did the pathway analysis; QX and TW performed the protein biochemistry, separations and mass spectrometry; HX constructed the hmuR mutant; $\mathrm{MH}$ and RJL conceived the experiments. $\mathrm{MH}$, ELH, MK and RJL wrote the manuscript. MK and ELH contributed equally. 


\section{Additional material}

\section{Additional file 1}

DataTables. Data tables, explanatory notes and supporting figures. This file contains the proteomic data tables ST1 and ST2, explanatory notes for each heading in the tables, a note regarding the handling of missing data and additional figures informative of proteome coverage for the model community described in data tables ST1 and ST2.

Click here for file

[http://www.biomedcentral.com/content/supplementary/14712180-9-98-S1.pdf]

\section{Acknowledgements}

This work was supported by NIDCR research grants DEI 4372, DEI 2505 and DEI I I I I, and by a Grant-in-Aid for Scientific Research (C)(20592453) from the Ministry of Education, Culture, Sports, Science and Technology of Japan. We thank the Institute for Systems Biology and Nittin Baliga for the use of Gaggle and assistance with the pathway analysis. We thank Fred Taub for the FileMaker database and assistance with the figures. We thank LANL (Los Alamos National Laboratory) and Gary Xie in particular for bioinformatics support.

\section{References}

I. Diaz PI, Chalmers NI, Rickard AH, Kong C, Milburn CL, Palmer RJ Jr, Kolenbrander PE: Molecular characterization of subject-specific oral microflora during initial colonization of enamel. Appl Environ Microbiol 2006, 72:2837-2848.

2. Rosan B, Lamont RJ: Dental plaque formation. Microbes Infect 2000, 2:1599-1607.

3. Ximenez-Fyvie LA, Haffajee AD, Socransky SS: Comparison of the microbiota of supra- and subgingival plaque in health and periodontitis. I Clin Periodontol 2000, 27:648-657.

4. Socransky SS, Haffajee AD, Ximenez-Fyvie LA, Feres M, Mager D: Ecological considerations in the treatment of Actinobacillus actinomycetemcomitans and Porphyromonas gingivalis periodontal infections. Periodontol 2000 1999, 20:34|-362.

5. Kolenbrander PE, Andersen RN, Blehert DS, Egland PG, Foster JS, Palmer RJ Jr: Communication among oral bacteria. Microbio Mol Biol Rev 2002, 66:486-505.

6. Kolenbrander PE, Palmer RJ Jr, Rickard AH, Jakubovics NS, Chalmers $\mathrm{NI}$, Diaz PI: Bacterial interactions and successions during plaque development. Periodontol 2000 2006, 42:47-79.

7. Marsh PD: Dental plaque as a biofilm and a microbial community - implications for health and disease. BMC Oral Health 2006, 6(Suppl I):S14.

8. Jenkinson HF, Lamont RJ: Oral microbial communities in sickness and in health. Trends Microbiol 2005, 13:589-595.

9. Whiteley M, Bangera MG, Bumgarner RE, Parsek MR, Teitzel GM, Lory S, Greenberg EP: Gene expression in Pseudomonas aeruginosa biofilms. Nature 200I, 4I 3:860-864.

10. Stoodley P, Sauer K, Davies DG, Costerton JW: Biofilms as complex differentiated communities. Annu Rev Microbiol 2002, 56:187-209.

II. Jakubovics NS, Gill SR, lobst SE, Vickerman MM, Kolenbrander PE: Regulation of gene expression in a mixed-genus community: stabilized arginine biosynthesis in Streptococcus gordonii by coaggregation with Actinomyces naeslundii. J Bacteriol 2008 190:3646-3657.

12. Simionato MR, Tucker CM, Kuboniwa M, Lamont G, Demuth DR Tribble GD, Lamont RJ: Porphyromonas gingivalis genes involved in community development with Streptococcus gordonii. Infect Immun 2006, 74:6419-6428.

13. Ang CS, Veith PD, Dashper SG, Reynolds EC: Application of 160 / 180 reverse proteolytic labeling to determine the effect of biofilm culture on the cell envelope proteome of Porphyromonas gingivalis W50. Proteomics 2008, 8:1645-1660.
14. Aas JA, Paster BJ, Stokes LN, Olsen I, Dewhirst FE: Defining the normal bacterial flora of the oral cavity. J Clin Microbiol 2005, 43:572I-5732.

15. Kuboniwa M, Tribble GD, James CE, Kilic AO, Tao L, Herzberg MC Shizukuishi S, Lamont RJ: Streptococcus gordonii utilizes several distinct gene functions to recruit Porphyromonas gingivalis into a mixed community. Mol Microbiol 2006, 60:121-139.

16. Lamont RJ, El-Sabaeny A, Park Y, Cook GS, Costerton JW, Demuth DR: Role of the Streptococcus gordonii SspB protein in the development of Porphyromonas gingivalis biofilms on streptococcal substrates. Microbiology 2002, I 48:1627-1636.

17. Capestany CA, Tribble GD, Maeda K, Demuth DR, Lamont RJ: Role of the Clp system in stress tolerance, biofilm formation, and intracellular invasion in Porphyromonas gingivalis. I Bacteriol 2008, 190:1436-1446.

18. Slots J, Gibbons RJ: Attachment of Bacteroides melaninogenicus subsp. asaccharolyticus to oral surfaces and its possible role in colonization of the mouth and of periodontal pockets. Infect Immun 1978, 19:254-264.

19. Bradshaw DJ, Marsh PD, Watson GK, Allison C: Role of Fusobacterium nucleatum and coaggregation in anaerobe survival in planktonic and biofilm oral microbial communities during aeration. Infect Immun 1998, 66:4729-4732.

20. Yao ES, Lamont RJ, Leu SP, Weinberg A: Interbacterial binding among strains of pathogenic and commensal oral bacterial species. Oral Microbiol Immunol I996, I I:35-4 I.

21. Foster JS, Kolenbrander PE: Development of a multispecies oral bacterial community in a saliva-conditioned flow cell. Appl Environ Microbiol 2004, 70:4340-4348.

22. Ebersole JL, Feuille F, Kesavalu L, Holt SC: Host modulation of tissue destruction caused by periodontopathogens: effects on a mixed microbial infection composed of Porphyromonas gingivalis and Fusobacterium nucleatum. Microb Pathog 1997, 23:23-32.

23. Saito A, Inagaki S, Kimizuka R, Okuda K, Hosaka Y, Nakagawa T, Ishihara K: Fusobacterium nucleatum enhances invasion of human gingival epithelial and aortic endothelial cells by Porphyromonas gingivalis. FEMS Immunol Med Microbiol 2008, 54:349-355.

24. Storey JD, Tibshirani R: Statistical significance for genomewide studies. Proc Natl Acad Sci USA 2003, 100:9440-9445.

25. Benjamini Y, Yekutieli D: Quantitative trait Loci analysis using the false discovery rate. Genetics 2005, I7I:783-790.

26. Storey Research Group, Qvalue [http://genomics.prince ton.edu/storeylab/qvalue/]

27. Xia Q, Hendrickson EL, Wang T, Lamont RJ, Leigh JA, Hackett M Protein abundance ratios for global studies of prokaryotes. Proteomics 2007, 7:2904-2919.

28. Knudsen S: Guide to analysis of DNA microarray data. Hoboken NJ: Wiley-Liss; 2004:33-55.

29. Hendrickson EL, Lamont RJ, Hackett M: Tools for interpreting large-scale protein profiling in microbiology. J Dent Res 2008 , 87:1004-1015.

30. Cleveland WS: A program for smoothing scatterplots by robust locally weighted regression. American Statistician 198I, 35:54

31. Naito M, Hirakawa $\mathrm{H}$, Yamashita A Ohara N, Shoji M, Yukitake $\mathrm{H}$, Nakayama K, Toh H, Yoshimura F, Kuhara S, et al.: Determination of the genome sequence of Porphyromonas gingivalis strain ATCC 33277 and genomic comparison with strain W83 revealed extensive genome rearrangements in $P$. gingivalis. DNA Res 2008, 15:215-225.

32. Xia Q, Wang T, Park Y, Lamont RJ, Hackett M: Differential quantitative proteomics of Porphyromonas gingivalis by linear ion trap mass spectrometry: non-label methods comparison, $q-$ values and LOWESS curve fitting. International Journal of Mass Spectrometry 2007, 259: 105-116.

33. Xia Q, Wang T, Taub F, Park Y, Capestany CA, Lamont RJ, Hackett M: Quantitative proteomics of intracellular Porphyromonas gingivalis. Proteomics 2007, 7:4323-4337.

34. Eng JK, McCormack AL, Yates JR: An approach to correlate tandem mass-spectral data of peptides with amino-acidsequences in a protein database. Journal of the American Society of Mass Spectrometry 1994, 5:976-989.

35. Chiu SW, Chen SY, Wong HC: Localization and expression of MreB in Vibrio parahaemolyticus under different stresses. Appl Environ Microbiol 2008, 74:7016-7022. 
36. Nomura M, Gourse R, Baughman G: Regulation of the synthesis of ribosomes and ribosomal components. Annu Rev Biochem 1984, 53:75-II7.

37. Schenk G, Duggleby RG, Nixon PF: Properties and functions of the thiamin diphosphate dependent enzyme transketolase. Int J Biochem Cell Biol 1998, 30: I 297-1318.

38. Roper JM, Raux E, Brindley AA, Schubert HL, Gharbia SE, Shah HN, Warren MJ: The enigma of cobalamin (Vitamin B I2) biosynthesis in Porphyromonas gingivalis. Identification and characterization of a functional corrin pathway. J Biol Chem 2000 , 275:403 I 6-40323.

39. Grenier D: Nutritional interactions between two suspected periodontopathogens, Treponema denticola and Porphyromonas gingivalis. Infect Immun 1992, 60:5298-530I.

40. Nelson KE, Fleischmann RD, DeBoy RT, Paulsen IT, Fouts DE, Eisen JA, Daugherty SC, Dodson RJ, Durkin AS, Gwinn M, et al.: Complete genome sequence of the oral pathogenic bacterium Porphyromonas gingivalis strain W83. J Bacteriol 2003, I 85:559|-560I.

4I. Volkert MR, Landini P: Transcriptional responses to DNA damage. Curr Opin Microbiol 200I, 4:I78-185

42. Lewis JP, Plata K, Yu F, Rosato A, Anaya C: Transcriptional organization, regulation and role of the Porphyromonas gingivalis W83 hmu haemin-uptake locus. Microbiology 2006 I 52:3367-3382.

43. Leveille S, Caza M, Johnson JR, Clabots C, Sabri M, Dozois CM: Iha from an Escherichia coli urinary tract infection outbreak clonal group A strain is expressed in vivo in the mouse urinary tract and functions as a catecholate siderophore receptor. Infect Immun 2006, 74:3427-3436.

44. Merritt J, Kreth J, Shi W, Qi F: LuxS controls bacteriocin production in Streptococcus mutans through a novel regulatory component. Mol Microbiol 2005, 57:960-969.

45. Washburn MP, Ulaszek R, Deciu C, Schieltz DM, Yates JR 3rd: Analysis of quantitative proteomic data generated via multidimensional protein identification technology. Anal Chem 2002, 74:1650-1657.

46. Washburn MP, Wolters D, Yates JR 3rd: Large-scale analysis of the yeast proteome by multidimensional protein identification technology. Nat Biotechnol 200I, 1 9:242-247.

47. Porphyromonas gingivalis W83 Genome Page [http:// cmr.jcvi.org/tigr-scripts/CMR/GenomePage.cgi?org=gpg]

48. Streptococcus gordonii Challis NCTC7868 Genome Page [http://cmr.jcvi.org/tigr-scripts/CMR/GenomePage.cgi?org=gsg]

49. Fusobacterium nucleatum ATCC 25586 Genome Page [http:/ /cmr.jcvi.org/cgi-bin/CMR/GenomePage.cgi?org=ntfn0I]

50. Mammalian Gene Collection [http://mgc.nci.nih.gov]

51. Peng J, Elias JE, Thoreen CC, Licklider LJ, Gygi SP: Evaluation of multidimensional chromatography coupled with tandem mass spectrometry (LC/LC-MS/MS) for large-scale protein analysis: the yeast proteome. J Proteome Res 2003, 2:43-50.

52. Elias JE, Gibbons FD, King OD, Roth FP, Gygi SP: Intensity-based protein identification by machine learning from a library of tandem mass spectra. Nat Biotechnol 2004, 22:2 I4-219.

53. Tabb DL, McDonald WH, Yates JR 3rd: DTASelect and Contrast: tools for assembling and comparing protein identifications from shotgun proteomics. J Proteome Res 2002, I:21-26.

54. Liu H, Sadygov RG, Yates JR 3rd: A model for random sampling and estimation of relative protein abundance in shotgun proteomics. Anal Chem 2004, 76:4193-4201.

55. Bosch G, Skovran E, Xia Q, Wang T, Taub F, Miller JA, Lidstrom ME, Hackett M: Comprehensive proteomics of Methylobacterium extorquens AMI metabolism under single carbon and nonmethylotrophic conditions. Proteomics 2008, 8:3494-3505.

56. Sokal RR, Rohlf FJ: Biometry, the principles and practice of statistics in biological research. New York: WH Freeman; 1995:715-724

57. Huang da W, Sherman BT, Tan Q, Kir J, Liu D, Bryant D, Guo $Y$, Stephens R, Baseler MW, Lane HC, et al.: DAVID Bioinformatics Resources: expanded annotation database and novel algorithms to better extract biology from large gene lists. Nucleic Acids Res 2007, 35:W169-175.

58. Aslanidis C, de Jong PJ: Ligation-independent cloning of PCR products (LIC-PCR). Nucleic Acids Res 1990, I 8:6069-6074.

59. Wu J, Lin X, Xie H: Regulation of hemin binding proteins by a novel transcriptional activator in Porphyromonas gingivalis. I Bacteriol 2009, 191: I 15-122.
60. O'Toole GA, Kolter R: Initiation of biofilm formation in Pseudomonas fluorescens WCS365 proceeds via multiple, convergent signalling pathways: a genetic analysis. Mol Microbiol I998, 28:449-46I.

6I. Capestany CA, Kuboniwa M, Jung IY, Park Y, Tribble GD, Lamont RJ: Role of the Porphyromonas gingivalis InIJ protein in homotypic and heterotypic biofilm development. Infect Immun 2006 , 74:3002-3005.
Publish with Biomed Central and every scientist can read your work free of charge

"BioMed Central will be the most significant development for disseminating the results of biomedical research in our lifetime. "

Sir Paul Nurse, Cancer Research UK

Your research papers will be:

- available free of charge to the entire biomedical community

- peer reviewed and published immediately upon acceptance

- cited in PubMed and archived on PubMed Central

- yours - you keep the copyright
BioMedcentral 